\title{
Pulsed Doppler echocardiography for diagnosis of ventricular septal defects
}

\author{
ANDREA MAGHERINI, GAETANO AZZOLINA, VIERI WIECHMANN, \\ FABIO FANTINI
}

From The Tuscan Heart and Chest Center, Florence, and the Department of Cardiology, University of Florence, Italy

SUMMARY In order to assess the performance of pulsed Doppler echocardiography and to examine the factors affecting its reliability for the diagnosis of ventricular septal defects, 51 patients, in whom angiographic studies had recently been performed, were investigated before and after operation by this technique. The diagnosis was correct in all cases with left-to-right shunt, when the defect was located either in the upper muscular or in the infracristal portion of the septum, even when pulmonary hypertension or additional cardiac malformations were present. Pulsed Doppler echocardiography did not differentiate between supracristal ventricular septal defects and obstruction of the right ventricular outflow. Apical muscular defects were never observed in our cases. Defects of the inlet portion were not localised by the method. In cases with bidirectional shunt, pulsed Doppler echocardiography failed to provide any diagnostic clue. When a right-to-left shunt was present, only in one case with membranous pseudoaneurysm could pulsed Doppler echocardiography provide an exact description of the haemodynamics before and after operation. In 12 of 22 patients with tetralogy of Fallot, pulsed Doppler echocardiography showed flow tracings possibly related to the septal defect. The site of defect and the extent and direction of intracardiac shunting seem to be the main factors affecting its diagnostic performance in ventricular septal defects.

Detection of ventricular septal defect by means of M-mode and/or bidimensional echocardiography is usually possible only when the defect is either large or associated with peculiar anatomical malformations, such as aortic dextroposition. Size and shape of the ventricular chambers and of the left atrium provide indirect information about the intracardiac haemodynamic situation. ${ }^{1}$ Bidimensional echocardiography can differentiate between ventricular septal defects and single ventricle complexes. ${ }^{2}$

Pulsed Doppler echocardiography, by providing information about the direction and the type (laminar vs. disturbed) of flow within the cardiac cavities and the great arteries, may prove to be a very sensitive diagnostic method for the diagnosis of ventricular septal defects. Encouraging results were reported in previous studies..$^{3-5}$

In this paper the data on preoperative angiocardiographic and echocardiographic examinations are compared with the description of the actual malformation, as observed at the time of cardiac surgery, and with the postoperative echocardiographic controls.

Received for publication 3 May 1979

\section{Subjects and methods}

Fifty-one patients, who had previously undergone cardiac catheterisation and who were scheduled for corrective open-heart surgery, were investigated by means of 'integrated echocardiography', including M-mode, bidimensional, and pulsed Doppler examinations, employing a wide-angle sector scanner (Varian V-3000) and a Doppler echocardiograph (ATL 500 A). The pulsed Doppler echocardiographic investigation was performed with the transducer applied on the praecordium. In order to recognise defects in different areas of the septum, the transducer was directed in the following way: (a) from the apex toward the aortic root along the echoes arising from the ventricular septum, as shown in the M-mode display, for identification of muscular or infracristal septal defects; (b) toward the tricuspid valve, to recognise defects located in the upper posterior aspect of the ventricular septum; and (c) rotating the transducer caudad, after recognition of the pulmonary valve, to identify supracristal defects.

In normal subjects, when the sample volume is 
located within the muscular septum, a low-pitched thumping sound can be heard. ${ }^{6}$ Time interval histogram and analogue display do not show any flow signal. Disturbed flow in this area, as indicated by a harsh. sound and by a wide dot pattern on the baseline, suggests shunting between the ventricles. ${ }^{7}$

Direct inspection of the heart at the time of operation confirmed the presence of a ventricular septal defect in all patients, either uncomplicated or associated with other malformations. In 15 patients the defect was uncomplicated, in two it was associated with an atrial septal defect, in two with tricuspid regurgitation, and in five with obstruction to the right ventricular outflow. In the remaining patients the ventricular septal defect was a component of a complex malformation: three patients were confirmed to have a complete atrioventricular canal, one a left ventricle to right atrium communication, one a membranous septal pseudoaneurysm, and 22 a tetralogy of Fallot.

Postoperatively, an integrated echocardiographic investigation was repeated to allow comparison of the new echocardiographic findings with those obtained before surgery.

\section{Results}

For better evaluation of our findings, three different intracardiac haemodynamic conditions will be considered separately.

(a) LEFT-TO-RIGHT SHUNTS

When the sample volume was located within the interventricular septum, a typical turbulence was recorded in all cases with defects of the muscular and infracristal type (Fig. 1). The defect was clearly identified by pulsed Doppler echocardiography even in six patients with pulmonary hypertension where the pulmonary artery pressure was higher than two-thirds of the systemic pressure. In three patients with a supracristal defect, pulsed Doppler echocardiography showed a systolic turbulence located in the right ventricular outflow tract, similar to that seen in cases with obstruction of the right ventricular outflow. When the septal defect was located in the inlet portion, as in the three patients with complete atrioventricular canal, systolic turbulence was detected behind the cusps of the tricuspid valve. In four patients with uncomplicated ventricular septal defect, where the pulmonary to systemic flow ratio was greater than 3 , mild systolic tubulence was detected in the right pulmonary artery, despite the absence of any obstruction of the right ventricular outflow. After surgical correction of the septal defect, these findings disappeared in all cases. ${ }^{9}$ Patients with ventricular septal defect and tricuspid regurgitation showed findings indicative of both the septal defect and valve regurgitation (Fig. 2). In the case of left ventricular right atrial communication a turbulent flow behind tricuspid valve echoes was recorded during systole.

\section{(b) BIDIRECTIONAL SHUNTS}

In two patients who had previously undergone a pulmonary artery banding, haemodynamic reevaluation showed a bidirectional shunt. The pulsed Doppler echocardiographic investigation did

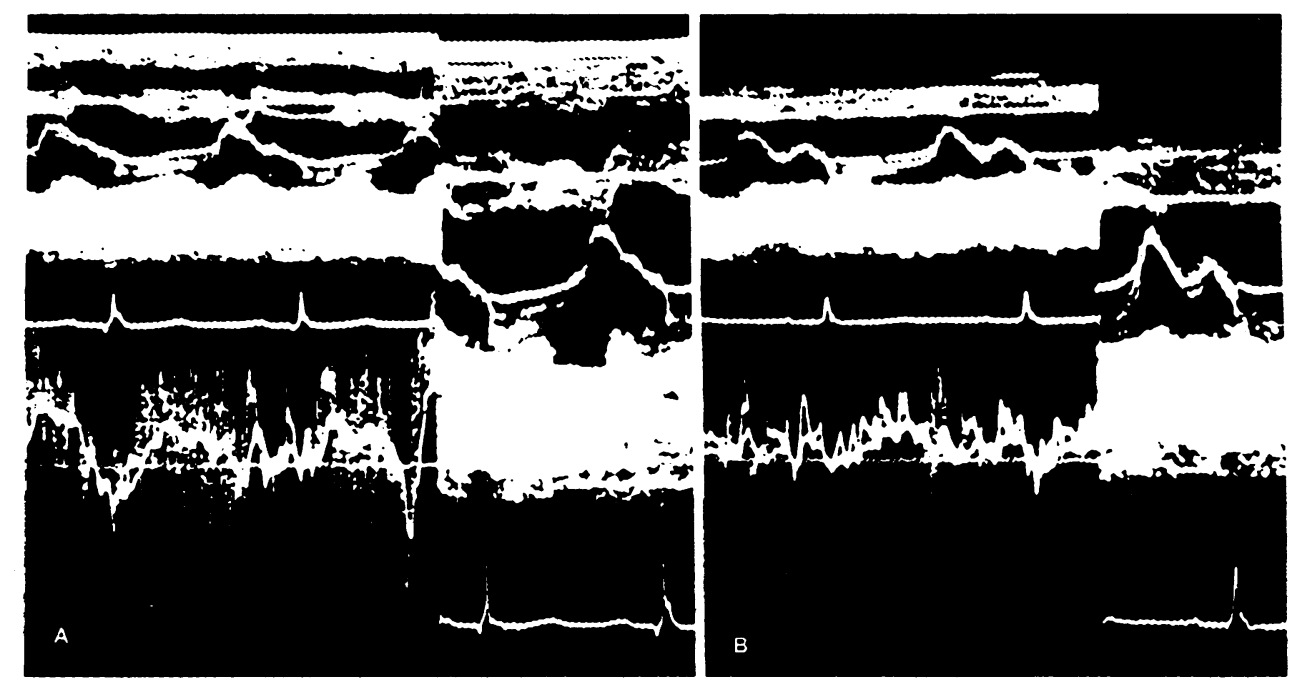

Fig. 1 Muscular ventricular septal defect: a systolic turbulence related to shunting is recorded (A), which is not detectable in the postoperative control $(\mathrm{B})$. 

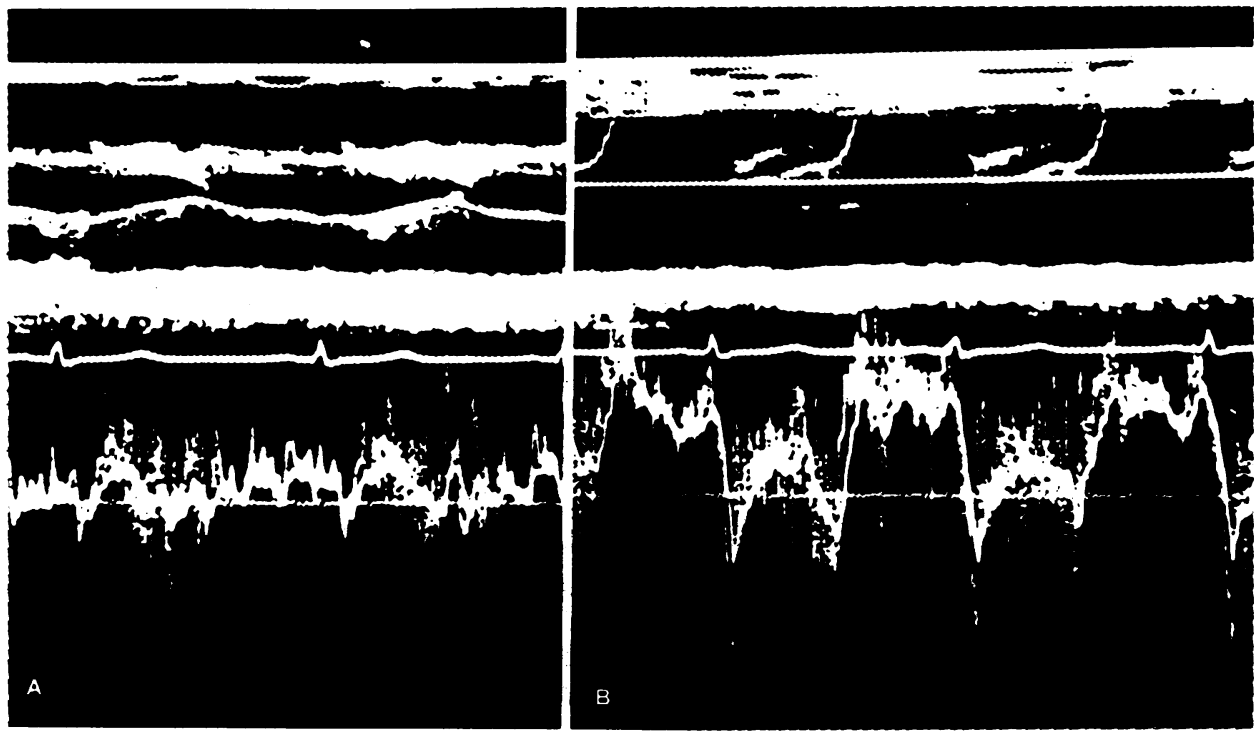

Fig. 2 Infracristal ventricular septal defect with tricuspid regurgitation: systolic turbulence is recorded within the septum, in the subaortic portion (A), and behind the anterior cusp of the tricuspid valve (B).

not provide any diagnostic clue in these cases, while bidimensional echocardiography detected the absence of a wide portion of the ventricular septum (Fig. 3). However, these two patients are not included in our series since their condition did not indicate corrective surgery.

(c) RIGHT-TO-LEFT SHUNTS

In 12 patients with tetralogy of Fallot, with the aorta fairly dextroposed, when the sample volume was located within the basal portion of the ventricular septum (as shown by $\mathbf{M}$-mode display) a systolic negative wave was recorded, indicating blood flowing away from the transducer (Fig. 4). In the remaining 10 patients in this group pulsed Doppler echocardiography did not provide any information related either to the location of the ventricular septal defect or to the direction of intracardiac shunting. In the patient with a pseudoaneurysm of the membranous septum and pronounced obstruction of the right ventricular outflow, pulsed Doppler echocardiography showed a distinct systolic turbulence when the sample volume was located in the area where $\mathrm{M}$-mode echocardiography detected an aneurysmal dilatation bulging toward the left ventricle in systole (Fig. 5). With the suprasternal approach a disturbed flow was detected in the ascending aorta. At surgical inspection, an accessory tricuspid cleft cusp was present, partially inserted onto the rim of the ventricular septal defect.

In all subjects, postoperative pulsed Doppler echocardiography showed the disappearance of the

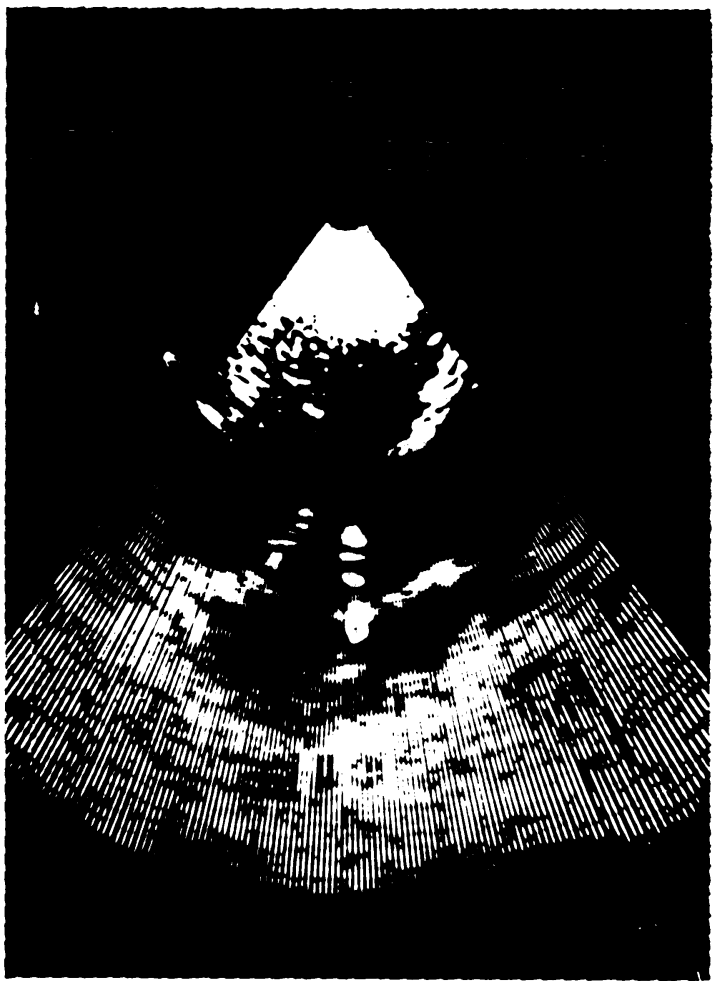

Fig. 3 Bidimensional image (apical approach) showing absence of an ample portion of the ventricular septum in a patient with bidirectional shunt. 


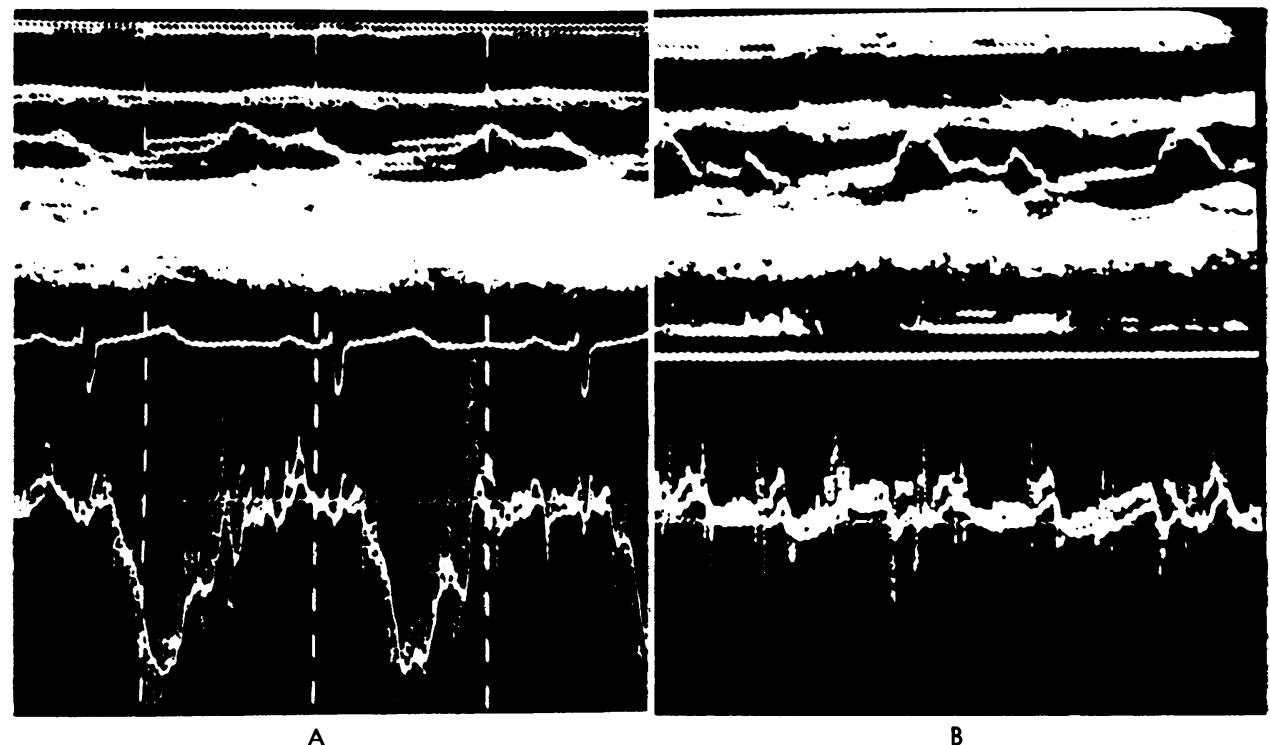

Fig. 4 Tetralogy of Fallot: a systolic negative deflection is detected in the preoperative ccntrol (A), which is not recorded postoperatively $(\mathrm{B})$.
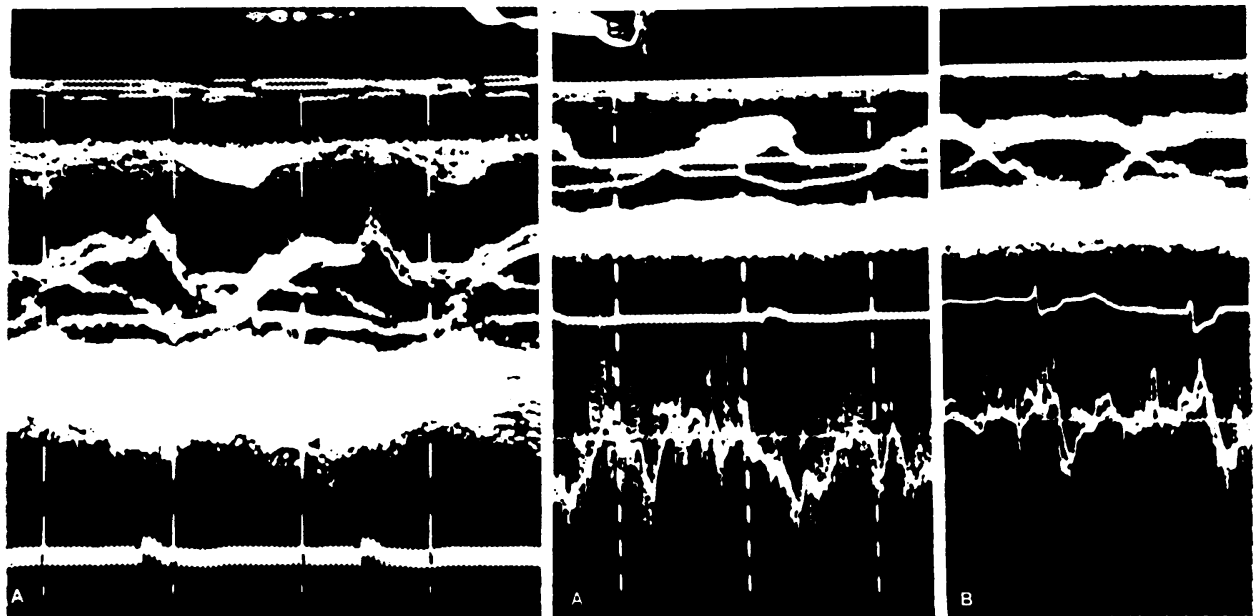

Fig. 5 Membranous pseudoaneurysm: systolic turbulence is recorded at the level of the septal aneurysmal bulging (A). Postoperative recordings show disappearance of both aneurysmal bulging and systolic turbulence (B).

preoperative findings related to ventricular septal defect.

\section{Discussion}

The site of the defect and the intracardiac haemodynamics are the main factors affecting the performance of pulsed Doppler echocardiography for the diagnosis of ventricular septal defects. In our experience, all patients with defects either of muscular or of infracristal type were correctly diagnosed by means of pulsed Doppler echocardiography even when pulmonary hypertension (with left-to-right shunt) or associated malformations (two patients with atrial septal defect, two with tricuspid regurgitation, three with right ventricular outflow obstruction) were present. Thus, our paper confirms the result of previous investigations. The typical diagnostic clue is the recording of a turbuletn flow when the sample vol ame is correctly located within the interventricular septum. The sign has to be highly specific: in our experience in both 


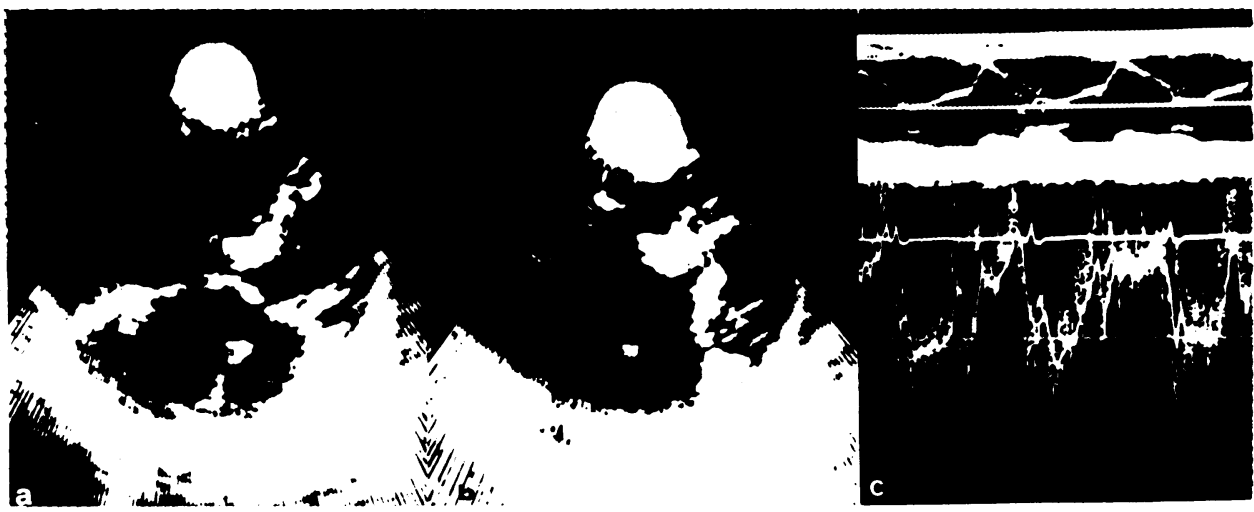

Fig. 6 Complete atrioventricular canal (Rastelli type A). Bidimensional image by the apical approach during systole (A) and diastole (B): systolic-diastolic turbulence is recorded behind the anterior cusp of the tricuspid valve (C) similar to that observed in one case of partial atrioventricular canal

normal subjects and in patients with various heart conditions it was never detected as an occasional finding. We did not see apical defects of the muscular septum during our study.

Supracristal, or distal infundibular, septal defects were characterised by a harsh systolic turbulence into the outflow tract of the right ventricle close to the echoes of the pulmonary valve. A similar finding was recorded in patients with obstruction of the right ventricular outflow: thus pulsed Doppler echocardiography does not allow differential diagnosis between the two conditions.

In patients with complete atrioventricular canal a systolic turbulence could be detected behind the echoes from the tricuspid valve (Fig. 6). We believe that this turbulent flow is related to shunting between the left ventricle and the right atrium through the mitral cleft, in the absence of the septum primum. In patients with atrial septal defects a turbulence behind the tricuspid valve is usually recorded at the telesystolic and protodiastolic phases.

In patients with complete atrioventricular canal the ventricular septal defect was clearly shown by bidimensional echocardiographic examination from the apical approach. Bidimensional echocardiography detected ventricular septal defects in patients with bidirectional shunt, while pulsed Doppler echocardiography did not provide any diagnostic information in these cases.

Bidimensional examination indicated the presence of ventricular septal defects in all patients with tetralogy of Fallot. In some of these cases pulsed Doppler echocardiography showed a blood flow toward the aortic outflow, through the basal portion of the septum, that might have been related to right-to-left shunting.

Our results suggest that pulsed Doppler echocardiography is an important complement of $\mathrm{M}$ mode and bidimensional echocardiography in the diagnosis of septal defects of both the upper muscular and the 'membranous' types. Further improvement of pulsed Doppler echocardiography will be achieved when technological developments allow location of the sample volume on a bidimensional display, thus enabling this diagnostic tool to be more widely used.

\section{References}

${ }^{1}$ Lewis AB, Takahashi $M$. Echocardiographic assessment of left to right shunt volume in children with ventricular septal defect. Circulation 1976; 54: 78-82.

${ }^{2}$ Silverman NH, Shiller NB. Apex echocardiography. A two-dimensional technique for evaluating congenital heart disease. Circulation 1978; 57: 503-11.

${ }^{3}$ Magherini A, Fantini F. L'ecocardiografia-doppler nella diagnosisi di alcune cardiopatie congenite. Atti III Congr. S.I.S.U.M. 1977; 257-62.

${ }^{4}$ Stevenson JG, Kawabori I, Guntheroth WG. Differentiation of ventricular septal defect from mitral regurgitation by pulsed doppler echocardiography. Circulation 1977; 56: 14-8.

${ }^{5}$ Stevenson JG, Kawabori I, Dooley T, Gunthreroth WG. Diagnosis of ventricular septal defect by pulsed doppler echocardiography - sensitivity, specificity, and limitations. Circulation 1978; 58: 322-6.

${ }^{6}$ Fantini F, Magherini A. Fonocardiografia intracavitaria per via incruenta mediante il Doppler ad onda pulsante. Atti III Congr. S.I.S.U.M. 1977: 253-6. ${ }^{7}$ Baker DW, Rubenstein SA, Lorch GS. Pulsed Doppler echocardiography: principles and applications. $\mathrm{Am} \mathcal{F}$ Med 1977; 63: 69-80.

${ }^{8}$ Lorch G, Rubenstein S, Baker D, Dooley T, Dodge H. Doppler echocardiography: use of a graphical display system. Circulation 1977; 56: 576-85.

${ }^{9}$ Magherini A, Fantini F. L'ecocardiografia-doppler nello studio del flusso transvalvolare polmonare. G Ital Cardiol 1978; 8: 520-7.

Requests for reprints to $\mathrm{Dr}$ Andrea Magherini, Tuscan Heart and Chest Center, Via Manzoni 12, 50121 Florence, Italy. 